\title{
Analysis of Digital Library Resource Discovery Service System
}

\author{
Zhi Gang Wang \\ Library of Yunyang Teachers' College, Shiyan, Hubei ,China, 442000 \\ Email:1244893215@qq.com
}

Keywords: Digital library; Resource discovery service; Retrieval; database; Electronic Resources

\begin{abstract}
This paper points out problems of traditional retrieval tools, introduces the background and development of researches on resource discovery service, focuses on analysis of its features and theoretical structure, and gives opinions on problems in the development of resource discovery service.
\end{abstract}

\section{Introduction}

Today, most libraries are built in a considerable scale by purchasing electronic resources or establishing database, and provide convenient information service for readers through Internet, which fully meets the readers' demand for information resources. Libraries have greater difficulties in electronic resource management, the workload is increasing, and readers are facing new problems. In addition, other information search engine providers such as Baidu and Google occupy a lot of reader resources due to convenient and easy advantages. These force the further integration of library resources so as to prevent library from being marginalized in the digital era. Facing the complex and changing information environment, how to integrate the existing library electronic resources and provide readers with an easy, unified and powerful platform for electronic resource is a problem that library technicians expect to solve. Digital resource discovery service provides an idea to solve this problem.

\section{Problems of Traditional Retrieval System}

Established in 2007, the Digital Library Federation (DLF) Integrated Library System (ILS) Discovery Interfaces Task Force (DITF) investigated the potential integration problems of relevant libraries between integrated library system and the application of external discovery tools ${ }^{[1]}$. The results showed that the current domestic library systems, including collection catalog OPAC and unified retrieval system, have the following problems:

a. The current OPAC retrieval system is developed for management of printed books and stacks. Its main function is to manage collected paper books, but it cannot integrate the increasing digital resources.

b. The current OPAC has simple compatible data standards and does not support catalog record function, which makes it difficult to be integrated with other database systems.

c. The current unified retrieval is only for electronic resources ordered by the libraries, but it cannot link Internet resources.

d. The interface of OPAC is not friendly enough. Readers are used to the easy and intuitive retrieval window of business search engine, so they are difficult to adjust to the powerful retrieval functions of OPAC, or they are easy to be confused.

e. OPAC system has defects in screening of search terms. It does not support correlation algorithm, it cannot correct wrong spell and it lacks semantic support.

f.The retrieval of OPAC depends on mechanical match too much. For known catalog information retrieval, if readers fail to give accurate titles, they cannot obtain desired results. 


\section{Resource Discovery Service and Its Features}

Research Status of Resource Discovery Service. Resource discovery service refers to a single search platform of simultaneously retrieving metadata of database and full data text. Different from general united retrieval, it does not conduct real-time external resource retrieval. Set from mechanism, the resource discovery service only retrieves metadata and full data text of the system, so its retrieval speed is faster. To some extent, resource discovery system is the evolution of unified retrieval. Some discovery service systems not only provide the integration of unified retrieval, but also use APT to retrieve information service from which the metadata cannot be obtained. The effect of this unified retrieval service is better than that of traditional discovery service.

At present, the platforms using resource discovery service mainly include ingentaconnect (http://www.ingentaconnect.com) and Infotrieve (mainly provide document retrieval and document delivery service) as well as Web of Science and Google Scholar. It provides a simple retrieval method to search academic literature in a wider range, and search out many disciplines and sources: academic press, professional team, data source prior to paper, academic reports of universities and other research institutes, thesis, book, abstract and text, and find research materials of the highest correlation from academic research in the world. These are resource discovery service platforms that have been put into use. In recent years, however, academic circles pay attention to research on resource discovery service that can integrate library OPAC as well as other digital information resources.

Features of Resource Discovery Service. According to Library Technology Reports issued in January 2011, the resource discovery service has the following four features ${ }^{[2]}$ :

a. Flexibility: compared with single database product or library OPAC, resource discovery service is not limited to single information database product. Its information service scope is wide and flexible and its flexibility space is greater.

b. Content: OPAC and electronic resource database are the main scope of resource discovery service, which establishes a content-rich database index according to some rules.

c. Transmission: when retrieving different databases, resource discovery service should rapidly and directly recommend diverse results to readers, and send the results that best meet the reader demands to readers according to some ordering rule.

d. Discovery: resource discovery service should support the retrieval requirement with simple retrieval condition as well as content recommendation related to retrieval term.

From the perspective of readers, the key element of resource discovery service is to enable readers to feel that the process from discovery to transmission is smooth and simple. Readers expect their retrieval platforms to provide a good experience similar to that of Google. Readers do not need to understand the difference between catalog and index abstract, and they even do not need to know whether the resources are in the catalog, electronic resource database provider, or free electronic resources. They do not expect to know these.

\section{Structure Indicators of Resource Discovery Service Retrieval System}

Advantages of Resource Discovery Service. Why does resource discovery system develop so fast and become a popular retrieval system among libraries? The reason is that it has the following three advantages.

a. Fast response speed: resource discovery system will establish an index database. Therefore, its retrieval is conducted in the index database without retrieval of metadata. Therefore, in terms of response speed, unified retrieval is obviously unmatched with resource discovery service.

b. Electronic resource database providers have the demand for improvement of retrieval system. More and more users of database hope to have simple retrieval experience at a fast response speed. Electronic resource database providers have the demand for cooperation with retrieval platform providers. 
c. Low cost: libraries use resource discovery service and unified retrieval, which can save costs.

System Structure. Resource discovery service uses unified index formats to retrieve all resources, while search engine only retrieves simple index database. Resource discovery service must handle database in an extremely different structure, and establish the simple index database after unified integration. In general, the retrieval system based on resource discovery service has the structure, shown in Fig.1.

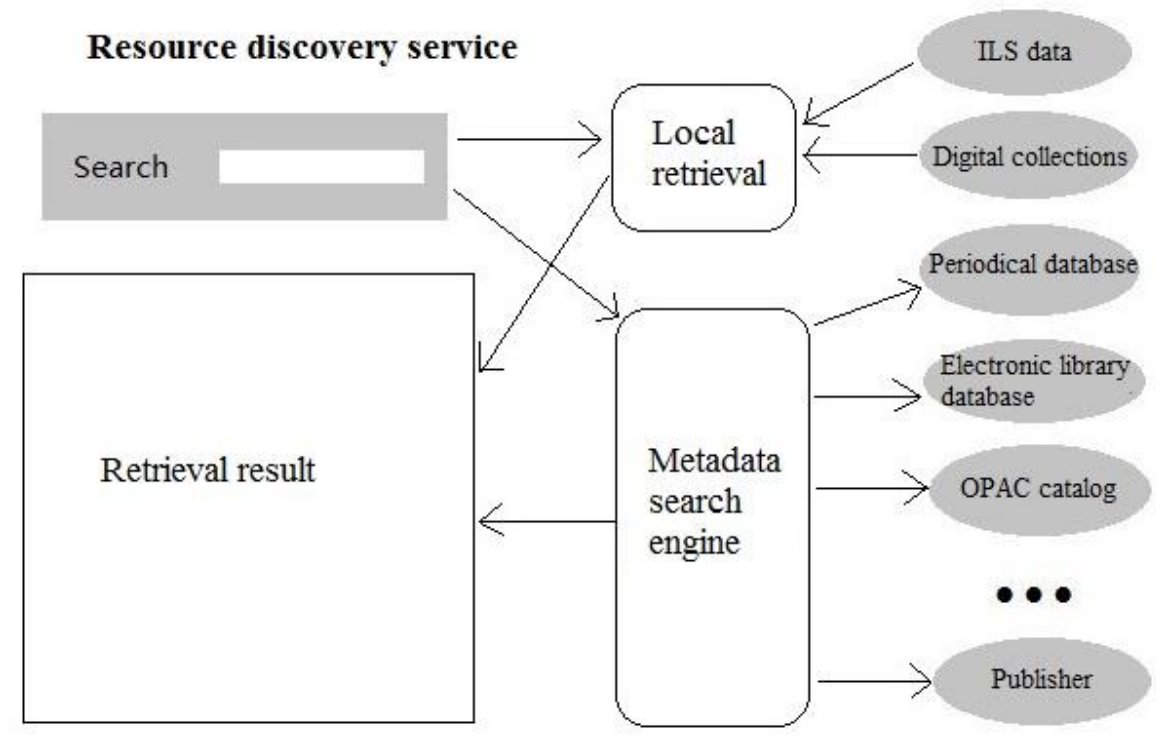

Figure. 1

Basic Indicators. Some basic indicators shall be followed in the establishment of retrieval system platform based on resource discovery service. These indicators include:

a. Content: depth and width of record content, richness and consistency of subsequently added data, frequency of content renewal, difficulty of subsequently added data for library catalog data and self-built database.

b. Retrieval function: easy and friendly retrieval interface, quality of retrieval results (accuracy according to order relevance), whether it can personalize retrieval results according to order relevance, whether it provides classification, layer analysis and other retrieval results navigation mechanism, integration of customer's current system (whether it can be integrated with OPAC and digital learning platform), whether it supports new operating environment (such as mobile end equipment). These are the indicators that measure retrieval function.

c. Consistency with library demands. Difficulty of system input, compatibility with current software and content, speed of response of manufacturers to reader needs and consultations, long and medium-term plan of subsequent development of system, overall evaluation of manufacturer by third parties. These are the important indicators that check whether the system meets library needs.

d. Price: price of system and maintenance fee. Many libraries are restricted by funds, especially local libraries and small and medium-sized libraries in universities. Whether the price is reasonable is an obstacle that affects the healthy development of resource discovery service.

\section{Shortcomings}

By establishing unified database index and integrating the unified retrieval interface, it can enable readers to quickly search paper books in OPAC library, digital resources provided by digital providers as well as open digital resources at a time. It orders the retrieval results according to relevance, displays them on the interface of resource discovery service retrieval system, and shows readers the required information. However, from the perspective of future, resource discovery service has the following problems in its development: 
a. Resource discovery service is only a convenient retrieval tool. Similar to traditional database retrieval, it can only retrieve e-books, thesis, e-newspaper and periodicals. There are few developers of sound, picture, video and similar information resources. It can only retrieve information resources of paper literatures in library, and the management still depends on traditional method.

b. The integration of Chinese data is more difficult. Not all traditional printed resources publishers and authors are willing to sell resources to electronic resource database manufacturers at a low price. The metadata is not easy to be authorized, which is bound to affect the using effect of library resource. Electronic resource database manufacturers should obtain the authorization of metadata as soon as possible.

c. The retrieval purpose of readers is to obtain the full text, while resource discovery service establishes a database index, which may not provide full text. Therefore, there will be a difference from reader needs and the retrieval experience may frustrate readers. Resource discovery service manufacturers should solve the problem.

d. Unified retrieval system has long been deployed by many libraries. Will library decision makers re-invest more funds in establishment of resource discovery service system? Obviously, this problem cannot be solved by library technicians, but completely depends on understanding and resolution of decision makers.

e. After the resource discovery service successfully integrates information resources of the library, readers will raise requirements for knowledge discovery system. Therefore, the integration of resource discovery service and library knowledge service system is a direction in the future.

The above problems hinder the development of resource discovery service. However, its effects have not been recognized as the resource discovery service has not been widely applied, so its development space is very large.

\section{References}

[1] Liang Fendong. Thinking of Past, Present and Future Trends of OPAC, J. Public Library Journal. ( 2010) 26-30.

[2] Chen Libing. Library Resource Discovery Service from the Perspective of Readers, J. Information and Documentation Services, (2012) 66-70.

[3] Xiang Ying, Li Xiangjun. Research on Application of Library Resource Discovery Service System in High Schools, J. Information Science, (2012) 1681-1684.

[4]Mohammad Norouzi Arab,Mohsen Sharifi. A model for communication between resource discovery and load balancing units in computing environments, J. The Journal of Supercomputing. (2014) 683.

[5]Mohammad Hasanzadeh,Mohammad Reza Meybodi. Distributed optimization Grid resource discovery, J. The Journal of Supercomputing, (2015) 711.

[6]Tinghuai Ma, Yinhua Lu,Sunyuan Shi,Wei Tian,Xin Wang,Donghai Guan. Data resource discovery model based on hybrid architecture in data grid environment, J. Concurrency Computat.: Pract. Exper. (2015) 273.

[7]Martha Cecilia Rosales-Herná,ndez,José, Correa-Basurto. The importance of employing computational resources for the automation of drug discovery, J. Expert Opinion on Drug Discovery, (2015) 103.

[8]Mahdi MollaMotalebi,Raheleh Maghami,AbdulSamad Ismail,Alireza Poshtkohi. THE EFFICIENCY CHALLENGES OF RESOURCE DISCOVERY IN GRID ENVIRONMENTS, J. Cybernetics and Systems, (2014) 458.

[9]Zhongping Zhang,Long He,Chao Zhang. Grid Resource Discovery Algorithm Based on Distance, 
J. Journal of Software, (2014) 911

[10]Cao Lijun,Liu Xiyin,Zhang Zhongping. Resource Discovery Algorithm Based on Multilayer Overlay Network, J. Journal of Networks,(2013) 86. 